\section{Features of endometrial cancer in patients with 'metabolically healthy' versus 'standard' obesity: the decreasing frequency of metabolically healthy obesity}

\begin{abstract}
Background: As endometrial cancer (EC) prevalence increases with obesity, we aimed to determine whether EC characteristics depend upon obesity type: 'standard' (SO) or 'metabolically healthy obesity' (MHO). Patients \& methods: 258 EC patients were included. Data on anthropometry, blood hormones, lipids and glucose, and tumor features were collected. Results: EC clinicopathologic characteristics and clinical stage correlate differently with BMI and obesity type. BMI is related inversely with tumor grade while SO patients are characterized by a more advanced clinical stage than those with MHO. Besides typical insulin resistance signs, EC patients with SO often display a higher serum leptin/adiponectin ratio compared with MHO patients. Historical data suggest a gradual increase in EC patient height and weight, and a decrease in $\mathrm{MHO}$ prevalence. Conclusion: It is currently unknown whether the latter observation reflects the evolution of EC, or obesity alongside the current epidemic. Regardless, the reduced MHO prevalence demonstrates the need for more intensive preventive measures aimed at obesity and obesity-associated conditions, including different EC subtypes.
\end{abstract}

Worldwide prevalence of uterine body or endometrial cancer (EC) has increased notably. Recently, the WHO documented an obesity epidemic in several countries. Concurrently, many studies showed a high (37-66\%) obesity rate in EC patients. There is currently no mention of a potentially distinctive correlation of EC with obesity types. In general, these types of obesity can be subdivided into 'standard' (SO; with metabolic disturbances, initially with insulin resistance signs) and 'nonstandard', or metabolically healthy obesity ( $\mathrm{MHO}$; without mentioned disturbances). The present paper concludes, first, that EC patients with SO (especially if BMI is $\geq 30$ ) generally have more advanced tumor stage than patients with $\mathrm{MHO}$. Second, in an EC group (2012-2014) a decrease in MHO frequency versus 1998-2000 group was observed. Altogether, this underlines the need for additional cancer preventive efforts in the obese female population.

Keywords: endometrial cancer $\bullet$ metabolically healthy obese $\bullet$ obesity types $\bullet$ tumor features and subtypes

The prevalence of malignant tumors as a rule increases with age. Some of these tumors occur most frequently in menopausal women when there is commonly an increase in body mass. Recently, the WHO documented an obesity epidemic in several countries $[1,2,3]$. While obesity is epidemic it is also highly heterogeneous $[4,5]$. This heterogeneity should be remembered while analyzing its epidemiology and impact on obesity-associated hormonedependent tumors, including endometrial cancer (EC).

Worldwide prevalence for $\mathrm{EC}$ has increased notably [6,7]. Concurrently, several metaanalyses conducted in Caucasian populations showed a high (37-66\%) obesity rate in EC
Lev M Berstein ${ }^{*, 1}$, Tatyana E Poroshina', Elena A Turkevich², Dmitry A Vasilyev ${ }^{1}$, Alexandra N Baltrukova ${ }^{3}$, Irina M Kovalenko' \& Igor V Berlev ${ }^{3}$ ${ }^{1}$ Laboratory of Oncoendocrinology, N.N. Petrov Research Institute of Oncology, St Petersburg 197758, Russia 2Department of Tumor Morphology, N.N. Petrov Research Institute of Oncology, St Petersburg 197758, Russia ${ }^{3}$ Division of Oncogynecology, N.N. Petrov Research Institute of Oncology, St Petersburg 197758, Russia *Author for correspondence: Tel.: +78124399536 Fax: +7 8125968947 levmb@endocrin.spb.ru 
patients $[8,9,10]$. The study of the prevalence and characteristics of EC and the correlation to obesity began long ago [11,12]. However, the data collected suggested discrepant opinions, especially on the association of obesity with EC features at diagnosis and the course of disease $[13,14,15,16,17]$.

These discrepancies can be explained in part by the commonplace evaluation of BMI instead of body fat content [3], a lack of clarity in regards to the actual role of steroidal (estrogens, progesterone, testosterone) and nonsteroidal (mostly insulin, leptin, adiponectin) components in obesity-cancer associations; and, a very probable and gradual evolution of EC characteristics themselves. Of note, this last point is mentioned infrequently but may play a significant role as evinced herein $[7,18]$. In addition to some other frequently ignored factors, one should note the rather peculiar biology of EC (making it strikingly different from other hormone-dependent cancers, e.g., breast). Furthermore, there is no mention of a potentially distinctive correlation of EC with obesity phenotypes $[4,5]$. In general, these phenotypes can be subdivided into 'standard' and 'nonstandard' categories.

According to modern concepts of obesity, most of its negative effects are due to combined adiposopathy (fat tissue function impairment) and insulin resistance (IR). The above mentioned obesity categories are distinguished mainly on whether IR is present and participates as a factor leading to profound alterations in hormone-metabolic processes ('standard') or not ('nonstandard'), while body fat content increases in both cases. The 'nonstandard' variant is often described as a 'metabolically healthy obesity' (MHO) state [19,20,21]. Mechanisms that could explain the more favorable metabolic profile of $\mathrm{MHO}$ individuals are poorly understood to date [21] and most aspects of how MHO relates to cancer development have yet to be studied $[22,23,24]$, which also is true for EC patients $[5,23]$.

As we reported previously, IR symptoms (independent of body fat content) correlate at the time of EC diagnosis with more advanced clinical stage and higher rates of local tumor invasion [25]. Whether this is true for obese patients without IR signs and associated hormone-metabolic features, remains unclear. It is important to stress here that the less favorable prognostic phenotype of treatment-naive EC is characterized commonly by higher HER-2/neu expression [26] and lower expression or mutation of the tumor suppressor PTEN [27] even though PTEN status remains a point of controversy [28]. Of note, there have been only a few studies on tumor PTEN expression in obese EC patients [29] where attempts to describe a correlation to obesity phenotypes were not made.
The current hospital based study was aimed at evaluating the association of BMI and obesity type ('standard' i.e., with IR signs, and 'nonstandard' or MHO) with important EC characteristics in treatment-naive patients studied in 1998-2000 and 2012-2014. In addition, the comparison of anthropometric parameters and the frequency of MHO in EC patients was made using data collected over the following periods: 1965-1967, 1998-2000 and 2012-2014.

\section{Patients \& methods}

A total of 258 EC patients were included on different stages of the study, which was approved by the local Ethics Committee and carried out in accordance with Declaration of Helsinki principles (2008 revision). With regards to the epidemiological design this was a 'descriptive study' where the source of population, that is patients with newly discovered EC, was all from the gynecological oncology clinic of N.N. Petrov Research Institute of Oncology. The usage of three time periods for patient evaluation (1965-1967; 1998-2000 and 2012-2014) was explained by the concentration of our group scientific interests on the problem of endometrial cancer just during those chronological phases of the last half-century and was justified by the presence of the same person (LMB) who participated in (1965-1967) or led (1998-2014) these studies.

The mean patient age was 60 years and this value was approximately the same when different time periods of patient data were compared. Data on histomorphological evaluation of the tumors are presented in the 'Results' section; in 2012-2014 around 77\% of the tumors were endometrioid adenocarcinomas. Body mass (in $\mathrm{kg}$ ) and height $(\mathrm{cm})$ of patients were measured in the Institute facility for arriving patients, and BMI was calculated as bodyweight $(\mathrm{kg}) /$ height $(\mathrm{m})^{2}$. The waist circumference was measured too. Correlation between BMI value and depth of tumor invasion (in $\mathrm{mm}$ ), differentiation (grade) and disease stage was evaluated for all patients (see the scale for clinical stage evaluation in Table 1), while fasting serum insulin levels and insulin resistance index (HOMA-IR [30]), were evaluated in patients $(n=192)$ admitted in 1998-2000 and 2012-2014. A total and visceral fat content was evaluated in last group of patients indirectly using bioelectrical impedance analysis (Tanita BC-543 Body Composition Monitor, Tokyo, Japan; manufactured in 2011). Serum insulin, leptin, estradiol, estrone and testosterone levels were evaluated by ELISA using kits from DRG Instruments $\mathrm{GmbH}$, Marburg, Germany, while adiponectin levels were determined with kits from DRG International Inc., Springfield Township, NJ, USA (with the day-today variance as a measure of analytical accuracy and parameter of quality control on the level 6.12, 6.43, 
5.19, 5.74, 5.33 and 6.72\%, respectively). Cholesterol, triglycerides and glucose in serum were evaluated by enzyme colorimetric assays using kits from Vector Best, Novosibirsk, Russia (day-to-day variance 3.69, 3.37 and $3.65 \%$, respectively). Serum samples from venous blood were obtained after overnight fasting.

In 70 randomly selected patients from the 2012-2014 group an additional immunohistochemistry assay for tumor tissue PTEN (mouse monoclonal 6H2.1) and HER-2/neu (c-erbB-2; polyclonal A0485) expression was performed using Dako Company (Glostrup, Denmark) antibodies. Epitope retrieval was performed with heating buffer DAKO TRS S1699, pH 6.0; the warm-up time/heating time was $30 \mathrm{~min}$. Antibodies were diluted 1:100 (PTEN) and 1:400 (HER-2/neu) by Dako antibody diluent. The incubation time was $30 \mathrm{~min}$ at $30^{\circ} \mathrm{C}$. Dako Real EnVision, HRP, rabbit/mouse K5007 (PTEN) and Dako EnVision, HRP Labelled polymer, antirabbit K4002 (HER-2/neu) were used as secondary antibodies (incubation time $30 \mathrm{~min}$, room temperature). The chromogen used was DAB in Dako Real Substrate buffer (all reagents from Dako Company, Glostrup, Denmark). See the 'Results' section for the criteria used to evaluate expression levels.

Patients admitted in 1998-2000 and 2012-2014 (192 pts total) were divided further into three groups based on BMI values: less than 25.0, 25.0-29.9 and $\geq 30.0$. Patients with $\mathrm{BMI}$ value of $\geq 25.0$ were evaluated additionally for obesity type (MHO or SO); see 'Discussion' section for explanation. Females who did not have a minimum three of the five following signs, based mostly on the criteria described in [31]: glucose tolerance impairment (fasting glucose $\geq 6.1 \mathrm{mmol} / \mathrm{l}$ ), hypertriglyceridemia $(\geq 1.7 \mathrm{mmol} / \mathrm{l})$, increased waist circumference $(\geq 88 \mathrm{~cm})$, low serum high-density cholesterol level $(\leq 1.3 \mathrm{mmol} / \mathrm{l})$ and hypertension $(\geq 130 / 85 \mathrm{~mm} \mathrm{Hg}$ ), were placed into the MHO group.

In patients with $\mathrm{MHO}$ and $\mathrm{SO}$ a comparative analysis of tumor characteristics, insulin level, insulin resistance index value (HOMA-IR calculated by the formula [glucose, $\mathrm{mmol} / \mathrm{l} \times$ insulin, $\mu \mathrm{U} / \mathrm{ml}$ ]/22.5]) and other parameters was performed based on the same principles as in BMI value-based groups. Additionally, data on the dynamics of anthropometric parameters and $\mathrm{MHO}$ prevalence in EC patients based on material collected by one of the authors (LMB) [32] for years 1965-1967 $(\mathrm{n}=66)$, then collected in 1998-2000 and processed by Kvatchevskaya $(n=74)$ [25], and finally, based on information obtained by us in 2012-2014 ( $\mathrm{n}=118)$ were evaluated and compared. This complex and 'extended' study structure is explained by its early date of initiation. These chronological periods were chosen, as said in the beginning of this section, because of the interest of the authors toward the problem of endometrial cancer and acquisition of the data in those time periods. This 'periodical' interest helped us assess whether endometrial cancer and patients with this tumor are same now as they were several decades ago. Obtaining informed consent was not mandatory for patients whose personal data were not recorded; therefore, it was sufficient to obtain an approval of the Local Ethics Committee to determine if an investigation corresponded to principles later formulated by the 2008 Declaration of Helsinki revision, which we strictly followed in the course of the study.

Statistical analysis was performed for this descriptive study using parametric and nonparametric methods. SigmaPlot for Windows and Statistica 8.0 software was used. Descriptive statistics were utilized to report demographic characteristics and anthropometrics. Variables were tested for normality using the Kolmogorov-Smirnov test. The comparison of hormone-metabolic values between different groups $(\mathrm{M} \pm \mathrm{SE})$ was performed by student's $t$-test. PTEN and HER-2/neu expression and clinical stage data were compared by $\chi^{2}$ test with one degree of freedom and Fisher's exact one-tailed test. The critical p-value throughout the whole study was 0.05 . The generalization of these findings (based on the studied sample) to a larger population will be the task for future studies.

Table 1. Features of the tumor and insulinemia in endometrial cancer patients (group of 2012-2014) depending on BMI.

\begin{tabular}{|c|c|c|c|c|c|c|c|}
\hline BMI grades & Age (years) & $\begin{array}{l}\text { BMI } \\
\text { (cond.un.) }\end{array}$ & $\begin{array}{l}\text { Differentiation } \\
\left.\text { (points }^{\dagger}\right)\end{array}$ & $\begin{array}{l}\text { Invasion } \\
(\mathrm{mm})\end{array}$ & $\begin{array}{l}\text { Clinical stage } \\
\text { (points }^{\ddagger} \text { ) }\end{array}$ & $\begin{array}{l}\text { Insulin } \\
(\mu \mathrm{U} / \mathrm{ml})\end{array}$ & $\begin{array}{l}\text { HOMA-IR } \\
\text { (cond.un.) }\end{array}$ \\
\hline$<25(n=17)$ & $59.4 \pm 2.7$ & $22.7 \pm 0.3^{*}$ & $2.07 \pm 0.18^{* * *}$ & $10.00 \pm 1.51$ & $2.66 \pm 0.36$ & $10.66 \pm 1.07 *$ & $2.85 \pm 0.37 *$ \\
\hline$\geq 30(n=73)$ & $61.1 \pm 1.0$ & $37.3 \pm 0.7^{*, * *}$ & $1.65 \pm 0.09 * * *$ & $7.68 \pm 0.82$ & $2.48 \pm 0.14$ & $21.10 \pm 1.14 * * *$ & $6.91 \pm 0.50^{*, * *}$ \\
\hline
\end{tabular}




\section{Results}

Higher BMI values were associated in EC patients with an increase in fasting serum insulin level and insulin resistance index. Additionally, the shift to more prognostically favorable variants of tumor differentiation, in agreement with data obtained previously [13,33], along with a tendency toward less evident myometrial invasion was revealed in patients with $\mathrm{BMI} \geq 30$. At the same time, no connection between BMI and clinical stage of cancer was found in EC patients (Table 1).

Although a probable shift with years in the distribution of EC morphologic variants and their correlation to anthropometry parameters will be considered separately in another publication, we would like to present some preliminary generalizations, which already can be made based on our data. In years 2012-2014 $77.2 \%$ of EC patients were diagnosed with endometrioid adenocarcinoma, while in the groups distinguished on the basis of BMI value (<25.0, 25.0-29.9 and $\geq 30.0$ ) this variant of the tumor was revealed in 70.6, 71.4 and $81.2 \%$ of cases, accordingly. In MHO and $\mathrm{SO}$ patients endometrioid adenocarcinoma was diagnosed in 77.7 and $78.5 \%$, respectively. In other words, an increase in BMI can be considered a 'modifying' factor in regard of tumor morphology, which was described previously [13,16], while obesity type cannot. Therefore, it was justified to examine whether EC patients belonging to $\mathrm{SO}$ and $\mathrm{MHO}$ groups and studied in 1998-2000 and 2012-2014 differed in other tumor characteristics and perhaps related hormonal characteristics.

A partial response to this question can be found in Table 2, which also contains respective data on nonobese (BMI <25.0) EC patients without metabolic changes. First, patients belonging to $\mathrm{MHO}$ group are younger and, as expected, have lower insulin resistance index and insulinemia values. Second, which is more related to the main goals of this study, although there was no difference in EC differentiation (clinical grade) and invasion depth between patients belonging to $\mathrm{SO}$ and MHO groups, SO patients were characterized by more advanced tumor stage, which was most evident in BMI $\geq 30$ group (Table 2). Of note, when insulin resistance index (HOMA-IR) as well as serum insulin, leptin and adiponectin levels were compared in EC patients, it was found that $\mathrm{SO}$ and $\mathrm{MHO}$ groups can be most effectively distinguished by HOMA-IR value and the ratio of leptin/adiponectin in serum. At the same time, serum leptin and adiponectin concentrations alone failed to demonstrate notable distinctions between these groups, especially when BMI value was $\geq 30$ (Table 3 ).

Serum estradiol and estrone levels were slightly higher in SO patients compared with patients with MHO (in relative values for $\mathrm{BMI} \geq 25$ group: 100 and $95.6 \%$, estradiol and 100 and $96.0 \%$, estrone), although in absolute values this difference was not statistically significant (data are not presented). The same conclusion is valid for blood testosterone levels,

Table 2. Features of the tumor and insulinemia in endometrial cancer patients (joint group of 1998-2000 and 2012-2014) depending on whether they belong to 'standard' or 'metabolically healthy' obesity subgroups.

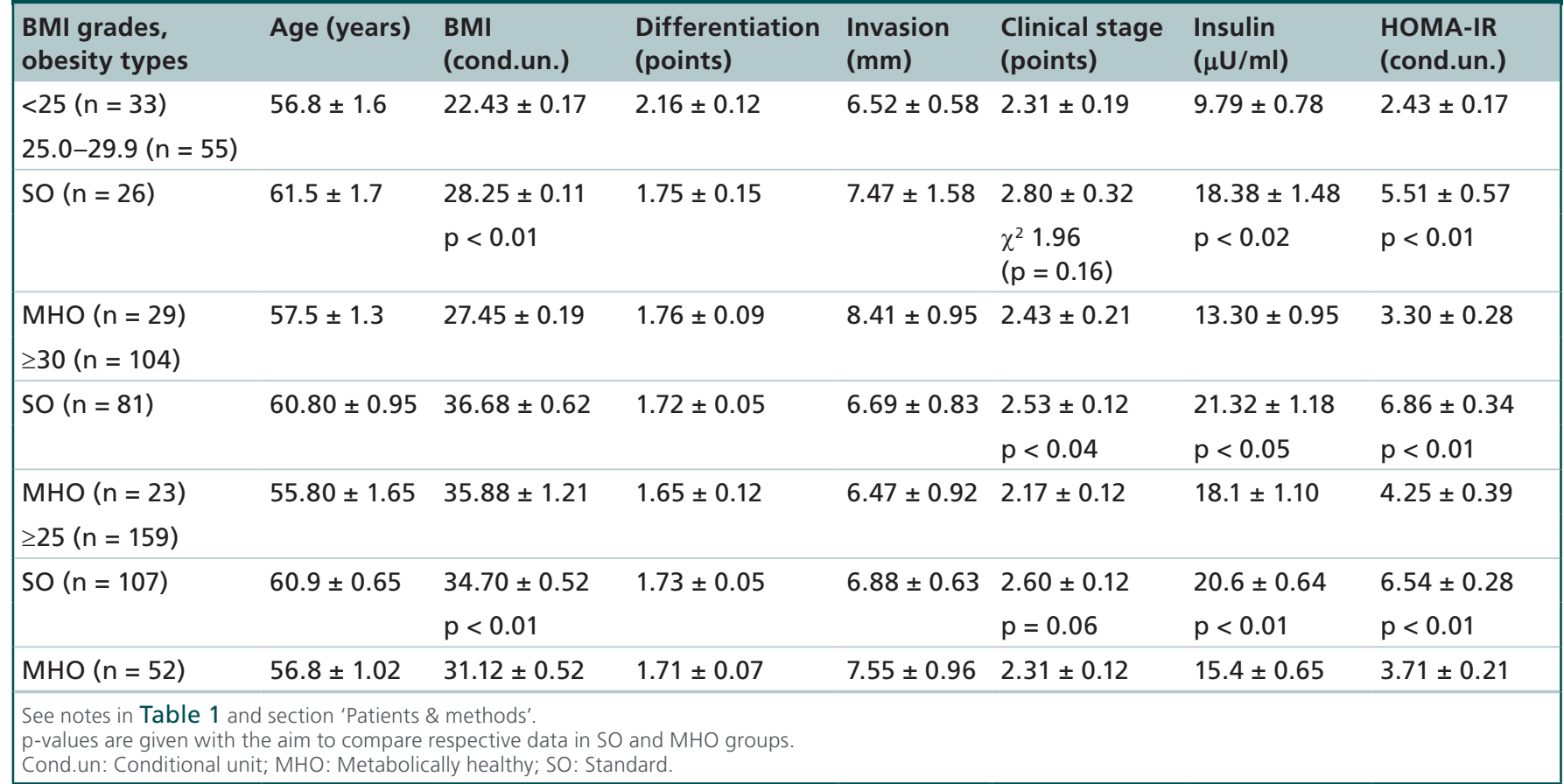


Table 3. Comparison of leptinemia, adiponectinemia and insulinemia levels in endometrial cancer patients (group of 2012-2014) depending on BMI value and obesity type.

\begin{tabular}{|c|c|c|c|c|c|c|}
\hline $\begin{array}{l}\text { BMI grades and types } \\
\text { of obesity }\end{array}$ & $\begin{array}{l}\text { BMI } \\
\text { (cond.un.) }\end{array}$ & L (ng/ml) & $\mathrm{A}(\mu \mathrm{g} / \mathrm{ml})$ & $\begin{array}{l}\text { Ratio L/A } \\
\text { (cond.un.) }\end{array}$ & $\begin{array}{l}\text { Insulin } \\
(\mu \mathrm{U} / \mathrm{ml})\end{array}$ & $\begin{array}{l}\text { HOMA-IR } \\
\text { (cond.un.) }\end{array}$ \\
\hline$<25(n=17)$ & $22.71 \pm 0.30$ & $5.23 \pm 0.77$ & $83.98 \pm 13.43$ & $0.11 \pm 0.04$ & $10.66 \pm 1.07$ & $2.85 \pm 0.37$ \\
\hline \multicolumn{7}{|l|}{$25.0-29.9(n=28)$} \\
\hline \multirow[t]{2}{*}{ SO $(n=13)$} & $29.01 \pm 0.19$ & $24.11 \pm 4.98$ & $39.44 \pm 9.84$ & $0.69 \pm 0.14$ & $21.89 \pm 2.79$ & $6.79 \pm 0.98$ \\
\hline & & $p=0.11$ & $p<0.02$ & $p>0.02$ & $p=0.06$ & $\mathrm{p}<0.02$ \\
\hline $\mathrm{MHO}(\mathrm{n}=15)$ & $28.03 \pm 0.35$ & $14.55 \pm 1.68$ & $75.92 \pm 10.72$ & $0.29 \pm 0.09$ & $15.80 \pm 1.61$ & $4.05 \pm 0.38$ \\
\hline \multicolumn{7}{|l|}{$\geq 30(n=73)$} \\
\hline \multirow[t]{2}{*}{ SO $(n=61)$} & $37.76 \pm 0.75$ & $33.71 \pm 3.14$ & $50.35 \pm 3.66$ & $0.91 \pm 0.16$ & $21.62 \pm 1.27$ & $7.37 \pm 0.56$ \\
\hline & & $p=0.09$ & & $p=0.07$ & $p>0.2$ & $p<0.02$ \\
\hline $\mathrm{MHO}(\mathrm{n}=12)$ & $34.95 \pm 1.53$ & $25.59 \pm 3.84$ & $49.05 \pm 5.42$ & $0.57 \pm 0.10$ & $18.40 \pm 2.41$ & $4.55 \pm 0.64$ \\
\hline \multicolumn{7}{|l|}{$\geq 25(n=101)$} \\
\hline \multirow[t]{2}{*}{ SO $(n=74)$} & $36.2 \pm 0.66$ & $31.88 \pm 2.75$ & $48.41 \pm 3.49$ & $0.87 \pm 0.14$ & $21.60 \pm 0.81$ & $7.28 \pm 0.28$ \\
\hline & & $p<0.01$ & $\mathrm{p}<0.05$ & $p<0.01$ & $p<0.01$ & $\mathrm{p}<0.01$ \\
\hline $\mathrm{MHO}(\mathrm{n}=27)$ & $31.12 \pm 0.52$ & $19.20 \pm 2.22$ & $64.60 \pm 7.18$ & $0.41 \pm 0.07$ & $17.00 \pm 1.03$ & $4.27 \pm 0.35$ \\
\hline
\end{tabular}

although EC patients with MHO, in contrast to estrogenemia, displayed values somewhat higher than in SO patients (Figure 1). Evaluation of total and visceral body fat content did not reveal a statistically significant increase in $\mathrm{SO}$ group compared with $\mathrm{MHO}$ patients except a total body fat value in patients with BMI 25.0-29.9: $\mathrm{SO} 42.43 \pm 0.79 \%$ versus $40.06 \pm 0.78 \%$ in $\mathrm{MHO}, \mathrm{p}=0.04$ and besides data in group of $\mathrm{EC}$ patients with $\mathrm{BMI} \geq 25.0$ (SO vs $\mathrm{MHO}$ : total fat content $46.13 \pm 0.59 \%$ and $42.06 \pm 0.83 \%, \mathrm{p}<0.01$; visceral fat $13.68 \pm 0.38 \%$ and $11.07 \pm 0.83 \%$, accordingly, $\mathrm{p}<0.01$ ).

Immunohistochemistry of EC markers (HER-2/neu and PTEN) was performed to correlate their expression with $\mathrm{BMI}$, and $\mathrm{SO}$ versus $\mathrm{MHO}$. Immunohistochemical evaluation of the tumor suppressor protein PTEN and the oncoprotein HER-2/neu in EC samples $(\mathrm{n}=70)$ revealed a U-shaped correlation between BMI and PTEN expression: the weakest expression was found in patients with a BMI between 25.0-29.9. Tumor HER-2/neu expression had an inverse relationship to BMI values: minimal expression in patients with a BMI $\geq 30$ (Table 4). At the same time, separate analysis of tumor tissue samples obtained from patients with distinct obesity phenotypes revealed the difference between groups. When obesity type was considered, a significant positive trend was found between BMI and PTEN expression in $\mathrm{MHO}$ group that does not exist in SO patients. In addition, tumors in $\mathrm{MHO}$ patients having BMI values between 25.0-29.9 displayed significantly lower HER-2/neu expression compared with nonobese patients (BMI <25.0), which also was not evident in tumors from $\mathrm{SO}$ patients. This demonstrates that besides a difference in clinical stage (Table 2) there are additional distinctions that exist at the tumor level between EC patients defined by different obesity phenotypes (Table 4).

Finally, the comparison of anthropometric features and $\mathrm{MHO}$ prevalence values in EC patients diagnosed in late 1960s (Period I), 1998-2000 (Period II) and 2012-2014 (Period III) discovered changes of studied parameters in the interval between Periods II and III. This observation manifested itself mostly in the form of pronounced body mass and height increases while the MHO frequency in Period III was lower (Figure 2). This was especially noted in patients having a BMI $\geq 30$ (not shown).

\section{Discussion}

There is evidence of an increase in the incidence of EC [6,7], which makes it not only a medical but also a social problem paralleling a similar situation in breast cancer [34]. The social significance of EC is explained largely by its relation to obesity, which has grown into a global epidemic [1,2]. Many researchers view obesity as an important contributor to the increase in the number of obesity-associated malignancies, including EC $[9,10,35]$, with some researchers convinced that obesity is a cause of EC (see [36]). EC patients as a rule are not losing weight, rather they are gaining weight. Indeed, most patients have been overweight or obese long before the diagnosis of endometrial cancer is made. Therefore, there is stronger reason to believe 


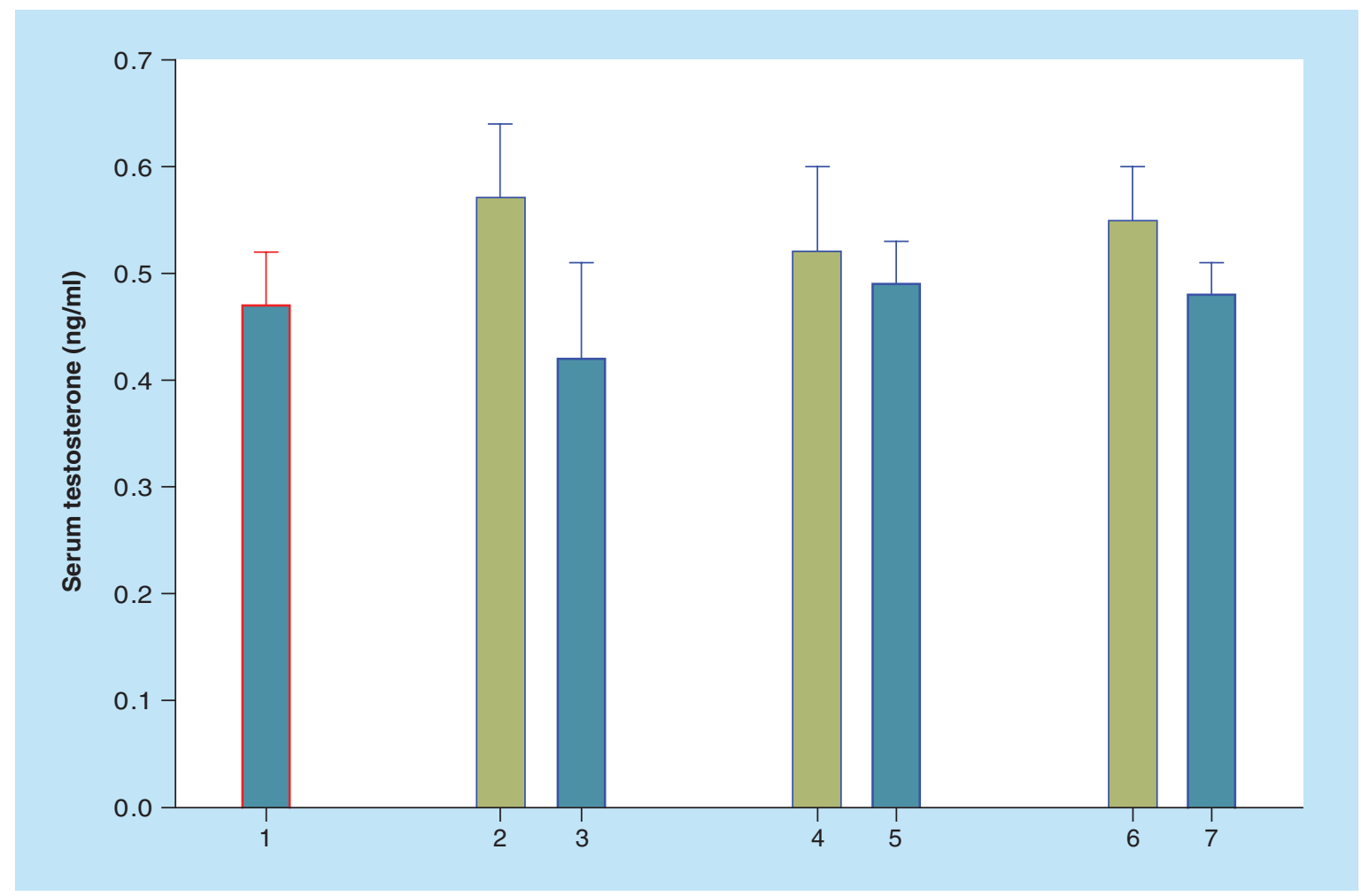

Figure 1. Serum testosterone level ( $\mathrm{ng} / \mathrm{ml}$; mean \pm standard error) in endometrial cancer patients. 1 : Patients with $\mathrm{BMI}<25.2$, 4, 6: Patients of MHO ('metabolically healthy obese') group with BMI values, respectively, 25.0-29.9, $\geq 30.0$ and $\geq 25.0$. 3, 5, 7: Patients of SO ('standard obesity') group with BMI values, respectively, 25.0-29.9, $\geq 30.0$ and $\geq 25.0$.

that BMI excess or obesity participate in shaping features of this cancer and not vice versa. Too frequently, however, there is a disregard with respect to the heterogeneous nature of obesity that influences many clinically relevant problems, including the problem of cancer in general and EC in particular. As a consequence, the notion that obesity is a heterogeneous condition [5] and EC-associated factor formed the basis of this study.

Coming to summation, we should first mention that the authors of this paper quite intentionally diverged from an accepted BMI-based obesity definition. As we know, according to WHO classification $\mathrm{BMI} \geq 25$ is considered as overweight and $\mathrm{BMI} \geq 30$ as obese. Nevertheless, in this investigation we included provisionally into the latter group the patients with both BMI values ( $\geq 30$ and $25.0-29.9$ ). The aim here was to examine how BMI value, on the one hand, and belonging to the $\mathrm{MHO}$ or $\mathrm{SO}$ group, on the other hand, is associated with clinical features of EC. Also, we wished to see whether there is marked discrepancy in this regard between what is defined by the terms 'obesity' and 'excessive bodyweight'. For a better overall understanding of the problem it is necessary to proffer several words expounding on the difference between 'standard obesity' and the notion of 'metabolic syndrome'. Although the role of metabolic syndrome in the development of EC has been studied and undoubtedly is important [18,25,36], one should note that BMI currently is not included in its official definition [37]. In addition, it is noteworthy that two types of EC have been discussed for several decades $[13,38]$. Current thought has these two types of EC characterized by a distinction in their etiology and risk factors [39], although such a conclusion has been questioned. Consequently, this leads to discussion of whether known causal factors are different for type I and type II EC or not [8,39]. Further, this brings to the forefront a discussion as to whether obesity plays the same role in both EC types $[6,8,10,16,33]$. On this basis, additional study of the problem and related subjects is warranted.

Thus, it is the strength of present study that obesity was not perceived as a singular entity. By separating obesity into $\mathrm{MHO}$ and $\mathrm{SO}$ groups, we revealed its different aspects in EC patients. Besides confirming a reverse correlation between BMI and tumor grade (Table 1), it allowed us to ascertain distinctions in certain tumor characteristics between patients with $\mathrm{SO}$ and $\mathrm{MHO}$, including disease stage (Table 2) and tumor-related protein expression (Table 4). Another strong point of this study is 
its 'historical/chronological view', which helped to discover the dynamics of anthropometrical characteristics and $\mathrm{MHO}$ frequency in EC patients, covering a time span of nearly 50 years (Figure 2). One could suppose that a gradual increase in the height of EC patients is just another confirmation of the acceleration phenomenon [40] according to which the 1910-1915 generation (at the end of 1960s they reached 55-60 years) might have lesser height than 1955-1960 generation (currently 55-60). At the same time, there is an evident trend toward increased body mass, which most probably reflects the ongoing development of the obesity epidemic [1,2] and correlates with the increases in EC incidence $[6,10,41]$.

This study also has some disadvantages or limitations; for example, we have omitted temporarily an analysis of EC subclassification based on tumor morphology and genetics $[8,39,42]$. As already mentioned, the relationship between EC morphology and obesity type ( $\mathrm{SO}$ or $\mathrm{MHO})$ is a matter for additional analysis since we completely understand that tumor morphology and subtype classification can change with years in different populations of patients [7]. Besides these points, while the study was hospital based and the sample size is relatively small (circa 260 patients), we need to stress that no special selection of the patients was made.
Another point for discussion lies in the nature of method used for body fat content determination. Although the method used (bioimpedance measurement) is indirect, it sufficed to fulfill the study objectives as the results of $\mathrm{SO}$ and $\mathrm{MHO}$ groups comparison appear to be quite accurate (see the 'Results' section). The usage of more sophisticated and simultaneously more expensive methods, like, for example, dual-energy $x$-ray absorptiometry and computed tomography in future studies will allow for comparative studies in this area to demonstrate suitability of bioimpedance under certain conditions [43] and to confirm received data.

The lack of conventional (common) definitions and criteria for MHO $[5,21,44]$ seems to be more important. According to the opinion of some experts the MHO state could be relatively stable over a 20区year perspective [45], and therefore, the term 'healthy' for this condition is justified only partly [46]. In addition, in the 1960s insulin levels and HOMA-IR values could not be determined, and therefore retrospective data were not available for EC patients from the early period. Nevertheless, as the most evident decrease in MHO prevalence was observed between 1998-2000 and 2012-2014 (Figure 2), in other words, when the signs of insulin resistance were taken into consideration, there is a basis to consider this observation as

\section{Table 4. PTEN и HER-2/neu expression in endometrial cancer tissue (immunohistochemical analysis).}

\begin{tabular}{|c|c|c|c|}
\hline BMI grades, number of cases & PTEN $^{+}$ & HER-2/neu ${ }^{\ddagger}$ & BMI (cond.un.) \\
\hline \multicolumn{4}{|c|}{ In patients with different BMI values: } \\
\hline$-<25.0(n=10)$ & $0.550 \pm 0.157$ & $1.333 \pm 0.167$ & $22.82 \pm 0.34$ \\
\hline$-25.0-29.9(n=19)$ & $0.417 \pm 0.109$ & $1.105 \pm 0.201$ & $28.45 \pm 0.27$ \\
\hline$-\geq 25.0(n=60)$ & $0.602 \pm 0.060$ & $0.867 \pm 0.102$ & $34.09 \pm 0.80$ \\
\hline$->30.0(n=41)$ & $0.683 \pm 0.069$ & $0.756 \pm 0.115$ & $36.71 \pm 0.91$ \\
\hline \multicolumn{4}{|c|}{ In patients with the signs of $\mathrm{MHO}$ : } \\
\hline$-<25,0$, without MHO $(n=10)$ & $0.550 \pm 0.157$ & $1.333 \pm 0.167^{3,4}$ & $22.82 \pm 0.34$ \\
\hline$-25.0-29.9(n=10)$ & $0.400 \pm 0.145^{1}$ & $0.900 \pm 0.314^{3}$ & $27.98 \pm 0.39$ \\
\hline$-\geq 25.0(n=18)$ & $0.555 \pm 0.114$ & $0.777 \pm 0.207$ & $30.99 \pm 1.23$ \\
\hline$-\geq 30.0(n=8)$ & $0.750 \pm 0.1641$ & $0.625 \pm 0.263^{4}$ & $34.75 \pm 2.10$ \\
\hline \multicolumn{4}{|l|}{ In patients with the signs of SO: } \\
\hline$-<25.0$; without SO $(n=10)$ & $0.550 \pm 0.157$ & $1.333 \pm 0.167^{5,6}$ & $22.82 \pm 0.34$ \\
\hline$-25.0-29.9(n=9)$ & $0.438 \pm 0.176^{2}$ & $1.333 \pm 0.236^{5}$ & $28.97 \pm 0.25$ \\
\hline$-\geq 25.0(n=42)$ & $0.622 \pm 0.072$ & $0.905 \pm 0.117$ & $35.42 \pm 0.95$ \\
\hline$-\geq 30.0(n=33)$ & $0.667 \pm 0.077^{2}$ & $0.788 \pm 0.129^{6}$ & $37.18 \pm 1.02$ \\
\hline \multicolumn{4}{|c|}{ 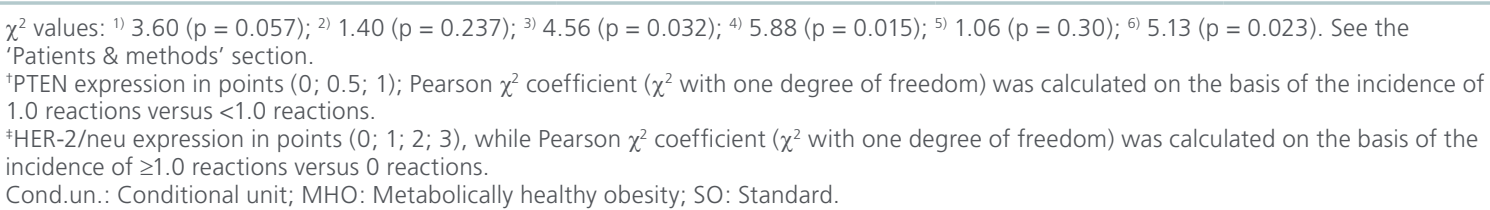 } \\
\hline
\end{tabular}




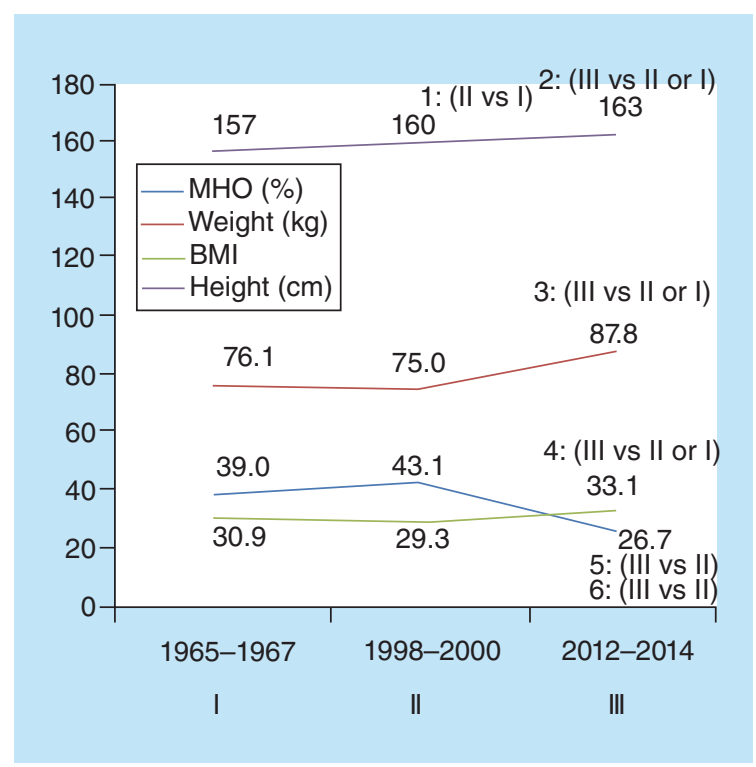

Figure 2. Comparison of anthropometric parameter values and the frequency of 'metabolically healthy obesity' in endometrial cancer patients evaluated in 1965-1967 (I), 1998-2000 (II) and 2012-2014 (III). $\mathrm{p}$-value according to the Student $t$-test: $1:<0.02$; $2:<0.01 ; 3:<0.01 ; 4:<0.01 ; 5:<0.05 ; 6:<0.02$. Presented data on $\mathrm{p}$-value are based each time on the comparison of two (and not multiple) groups. For example, $p$ for height is calculated for the pair 'Period II/Period I' or 'Period III/Period II', and so on.

important and reflecting not only on the evolution of obesity but the evolution of EC itself too $[7,18,47]$, see additionally below.

The progression to a more advanced EC clinical stage in patients with $\mathrm{SO}$ (Table 2) might be promoted by higher proportions of total and visceral body fat content, severe insulin resistance, higher serum leptin/adiponectin ratio and probably the mild prevalence of estrogenemia but not testosteronemia as compared with patients with MHO (Tables 1-3 \& Figure 1). Here we briefly mention the following supportive observations. First of all, leptin and adiponectin receptor density in EC tumor tissue correlates directly for leptin and inversely for adiponectin receptors, with regional lymph nodes involvement $[48,49]$. On the other hand, total serum testosterone levels tend to be higher in patients with EC [50] as it occurs in postmenopausal women with developing insulin resistance [51]. This is contrary to men where the resistance to insulin is more often the sign of low androgen level [52]. Meanwhile, there is no data to date on testosterone levels in patients with $\mathrm{MHO}$ and our preliminary results that unexpectedly indicated mildly lower testosteronemia in patients with SO compared with patients with MHO (Figure 1) deserve further verification.

No less than one third of patients admitted in 2012-2014 had signs of Type 2 diabetes mellitus
(DM2) discovered due to evaluation of the case histories or revealed during glucose tolerance test. Accordingly, the issue whether the combination of different variants of obesity, $\mathrm{SO}$ or, understandably much less frequent, MHO, and DM2 has any 'specific' influence on tumor biology in EC patients warrants special study, since to date this problem was studied only as applied to obesity en masse $[13,53]$ and was concentrated so far mostly on peripheral regulatory pathways. Furthermore, most of these studies were done without evaluation of PTEN and HER-2/neu expression in endometrial tumor tissue. This provides a topic for further comparative studies in EC patients with or without DM2 as we have shown altered expression of these markers among the obesity subtypes, $\mathrm{SO}$ versus $\mathrm{MHO}$, in EC patients.

Of note, although the tumor suppressor protein PTEN expression was mentioned just above in the context of tumor tissue, in mouse models where additional copies of PTEN are expressed, the mice have a longer life span, lower frequency of spontaneous tumors and an increase in brown adipose tissue [54]. According to published data, which are not completely consistent, the quantitative and functional characteristics of brown adipose tissue may be used as an indicator of a predisposition to obesity [55,56,57] and one of the markers of its heterogeneity $[5,58]$. This aspect of the problem also has not been studied in EC patients, although it undoubtedly presents scientific and practical interest and deserves attention. The understanding of importance of distinctive obesity phenotypes and their role in EC pathogenesis and clinical course can lead also to better utilization and implementation of treatments derived from adipose stem cells and factors secreted by them [59].

\section{Conclusion}

The main results of this study are summarized in Tables 1-4 \& Figures 1 \& 2. Based on these data one can jump to the following conclusions. First, some endometrial cancer characteristics, such as differentiation, depth of myometrial invasion, clinical stage and expression of PTEN and HER-2/neu by tumor tissue are correlated to a variable degree with BMI value and obesity type ('metabolically healthy obesity' or 'standard obesity'). In particular, it is rather well known $[13,33]$ that there is better tumor differentiation in patients with increased BMI (Table 1), while, after 'obesity partition', the disease stage appears to be more advanced in EC patients with combination of $S O$ and $\mathrm{BMI} \geq 30$ in contrast to patients with $\mathrm{MHO}$ and same BMI value (Table 2). Second, we compared historical data that provide evidence of progressive change in mean height and weight of endometrial cancer patients. These 
parameters are gradually increasing over time while, on the other hand, $\mathrm{MHO}$ is now encountered more rarely in the contemporary EC population (Figure 2). Therefore, the actual and anticipated increase in EC incidence may depend not only on the fast growth of the obesity epidemic, but also on changes in obesity structure and, presumably, in white/brown adipose tissue interrelations. As a whole, this may require reviewing and changing certain measures aimed at the prevention of obesity and obesity-associated conditions including uterine body cancer.

\section{Disclosure}

The data were presented in part in poster form at 97th Endocrine Society (USA) Meeting on 5 March, 2015: LM Berstein, DA Vasilyev, IV Berlev, TE Poroshina 'Metabolically Healthy Obesity: Changing Prevalence in Endometrial Cancer and Relation with Clinical Features of This Tumor' (THR-577).

\section{Author contributions}

Conceived and designed the study: LM Berstein, collected and evaluated data: LM Berstein, TE Poroshina, EA Turkevich, DA Vasilyev, AN Baltrukova, IM Kovalenko, IV Berlev. Draft of the paper was written by LM Berstein. Revised the manuscript: LM Berstein, TE Poroshina, EA Turkevich, DA Vasilyev, AN Baltrukova, IM Kovalenko, IV Berlev. Final version of the paper was read and approved by LM Berstein, TE Poroshina, EA Turkevich, DA Vasilyev, AN Baltrukova, IM Kovalenko, IV Berlev.

\section{Acknowledgements}

The authors are grateful to RA Sikes (Center for Translational Cancer Research, Department of Biological Sciences University of Delaware, Newark, DE, USA) and A Shpakov
(Laboratory of Molecular Endocrinology and Neurochemistry, Institute of Evolutionary Physiology and Biochemistry of RAS, St Petersburg, Russia) for their helpful and fruitful discussions.

Financial \& competing interests disclosure

The study was partly supported by grant of Russian Foundation of Basic Research (15-04-00384; LM Berstein, TE Poroshina, DA Vasilyev, IM Kovalenko; all parts of the study) and Russian Scientific Foundation (No 14-15-00413; LM Berstein, IM Kovalenko; evaluation of the data on the prevalence of diabetes in endometrial cancer patients and on potential role of diabetes combination with obesity). The authors have no other relevant affiliations or financial involvement with any organization or entity with a financial interest in or financial conflict with the subject matter or materials discussed in the manuscript apart from those disclosed.

No writing assistance was utilized in the production of this manuscript.

\section{Ethical conduct of research}

The authors state that they have obtained appropriate institutional review board approval or have followed the principles outlined in the Declaration of Helsinki for all human or animal experimental investigations. In addition, for investigations involving human subjects, informed consent has been obtained from the participants involved.

\section{Open access}

This work is licensed under the Creative Commons Attribution 4.0 License. To view a copy of this license, visit http:// creativecommons.org/licenses/by/4.0/

\section{Executive summary}

- Obesity can be divided into 'standard obesity' (SO; characterized by insulin resistance and associated hormone-metabolic changes) and nonstandard ('metabolically healthy obesity' [MHO]); this classification seems to be justified for the area of endometrial cancer (EC) research, although the optimal MHO definitions and criteria are still to be discussed.

- EC patients with SO (especially if $\mathrm{BMI}$ is $\geq 30$ ) generally have more advanced disease and a tendency to higher HER-2/neu expression in tumor tissue in contrast to patients with MHO.

- EC patients with SO are characterized by some additional hormonal factors including a marked increase in the ratio of serum leptin/adiponectin and an 'unexpected' tendency to mildly lower testosteronemia compared with patients with $\mathrm{MHO}$.

- The analysis of anthropometric characteristics of EC patients over the last 40-50 years in the same hospital discovered a gradual increase of height, weight and BMI in this population. In EC group of 2012-2014 a decrease in MHO frequency was observed. As a whole, this can be considered as a marker of the shifts in obesity epidemic parameters, both quantitative as well as qualitative, which also could influence other, gradually accumulating, manifestations of endometrial carcinoma evolution.

- Heterogeneity of obesity in combination with growing heterogeneity of endometrial cancer (evident, e.g., in the change of the ratio [7] and number [60] of EC subtypes) is a factor in favor of the development of additional preventive and control measures in accord with the title of the recent publication in this area: "Catch it before it kills..." [61]. 


\section{References}

Papers of special note have been highlighted as:

- of interest; $\bullet \bullet$ of considerable interest

1 James WP. WHO recognition of the global obesity epidemic. Int. J. Obes. (Lond.). 32(Suppl. 7), S120-S126 (2008).

2 Mitchell S, Shaw D. The worldwide epidemic of female obesity. Best Pract. Res. Clin. Obstet. Gynaecol. 29(3), 289-299 (2014).

3 Pasco JA, Holloway KL, Dobbins AG, Kotowicz MA, Williams LW, Brennan SL. Body mass index and measures of body fat for defining obesity and underweight: a cross-sectional, population-based study. BMC Obesity doi:10.1186/2052-9538-1-9 (2014) (Epub ahead of print).

4 Bray GA. Evaluation of obesity. Who are the obese? Postgrad. Med. 114(6), 19-27 (2003).

5 Berstein LM. Cancer and heterogeneity of obesity: a potential contribution of brown fat. Future Oncol. 8(12), 1537-1548 (2012).

6 Sheikh MA, Althouse AD, Freese KE et al. USA Endometrial Cancer Projections to 2030: should we be concerned? Future Oncol. 10(16), 2561-2568 (2014).

- Very important paper that shows unfavorable tendencies in endometrial cancer incidence and describes potential causes of this including the role of obesity.

7 Evans T, Sany O, Pearmain P et al. Differential trends in the rising incidence of endometrial cancer by type: data from a UK population-based registry from 1994 to 2006. Br. J. Cancer 104(9), 1505-1510 (2011).

8 Setiawan VW, Yang HP, Pike MC et al. Type I and II endometrial cancers: have they different risk factors? J. Clin. Oncol. 31(20), 2607-2018 (2013).

9 Zhang Y, Liu H, Yang S, Zhang J, Qian L, Chen X. Overweight, obesity and endometrial cancer risk: results from a systematic review and meta-analysis. Int. J. Biol. Markers 29(1), e21-e29 (2014).

10 Renehan AG, Soerjomataram I, Leitzmann MF. Interpreting the epidemiological evidence linking obesity and cancer: a framework for population-attributable risk estimations in Europe. Eur. J. Cancer 46(14), 2581-2592 (2010).

11 Twombly GH, Scheiner S, Levitz M. Endometrial cancer, obesity, and estrogenic excretion in women. Am. J. Obstet. Gynecol. 82, 424-427 (1961).

12 Elwood JM, Cole P, Rothman KJ, Kaplan SD. Epidemiology of endometrial cancer. J. Natl Cancer Inst. 59 (4), 1055-1060 (1977).

13 Bokhman JV. Two pathogenetic types of endometrial carcinoma. Gynecol. Oncol. 15(1), 10-17 (1983).

14 Arem H, Irwin ML. Obesity and endometrial cancer survival: a systematic review. Int. J. Obes. (Lond.) 37(5), 634-639 (2013).

15 Crosbie EJ, Roberts C, Qian W et al. Body mass index does not influence post-treatment survival in early stage endometrial cancer: results from the MRC ASTEC trial. Eur. J. Cancer 48(6), 853-864 (2012).

16 Fader AN, Arriba LN, Frasure HE, von Gruenigen VE. Endometrial cancer and obesity: epidemiology, biomarkers, prevention and survivorship. Gynecol. Oncol. 114(1), 121-127 (2009).

17 Lino-Silva LS, de León DC, Salcedo-Hernández RA et al. A high body mass index is not a worse prognostic factor for endometrial carcinoma in a predominantly obese population. Clin. Transl. Oncol. 15(3), 243-247 (2013).

18 Bershte n LM. [Endometrial cancer, estrogens and metabolic syndrome: scenario becomes more complicated]. Vopr. Onkol. 60(3), 254-262 (2014).

19 Sims EA. Are there persons who are obese, but metabolically healthy? Metabolism 50, 1499-1504 (2001).

-• Paper with a big potential, which initiated contemporary interest to the problem of metabolically healthy obesity.

20 Karelis AD. Metabolically healthy but obese individuals. Lancet 372(9646), 1281-1283 (2008).

21 Primeau V, Coderre L, Karelis AD et al. Characterizing the profile of obese patients who are metabolically healthy. Int. J. Obes. (Lond.) 35(7), 971-981 (2011).

22 Calori G, Lattuada G, Piemonti L. Prevalence, metabolic features, and prognosis of metabolically healthy obese Italian individuals: the Cremona Study. Diabetes Care 34(1), 210-215 (2011).

- One of the first papers that attracted attention to the possibility of differing influence of metabolically healthy obesity on cancer outcomes.

23 Moore LL, Chadid S, Singer MR, Kreger BE, Denis GV. Metabolic health reduces risk of obesity-related cancer in Framingham study adults. Cancer Epidemiol. Biomarkers Prev. 23(10), 2057-2065 (2014).

24 Oh CM, Jun JK, Suh M. Risk of cancer mortality according to the metabolic health status and degree of obesity. Asian Pac. J. Cancer Prev. 15(22), 10027-10031 (2014).

25 Berstein LM, Kvatchevskaya JO, Poroshina TE et al. Insulin resistance, its consequences for the clinical course of the disease, and possibilities of correction in endometrial cancer. J. Cancer Res. Clin. Oncol. 130(11), 687-693 (2004).

26 Kudela M, Pilka R, Lubusky M, Hejtmanek P, Dzubak P, Brychtova S. Prognostic importance of selected molecular immunohistochemical markers and DNA ploidy in endometrial cancer. Eur. J. Gynaecol. Oncol. 33(2), 159-163 (2012).

27 Catasus L, Gallardo A, Cuatrecasas M, Prat J. Concomitant PI3K-AKT and $\mathrm{p} 53$ alterations in endometrial carcinomas are associated with poor prognosis. Mod. Pathol. 22(4), 522-529 (2009).

28 Akiyama-Abe A, Minaguchi T, Nakamura Y. Loss of PTEN expression is an independent predictor of favourable survival in endometrial carcinomas. Br. J. Cancer 109(6), 1703-1710 (2013).

29 Dellas A, Jundt G, Sartorius G, Schneider M, Moch H. Combined PTEN and $\mathrm{p} 27 \mathrm{kip} 1$ protein expression patterns are associated with obesity and prognosis in endometrial carcinomas. Clin. Cancer Res. 15(7), 2456-2462 (2009).

30 Matthews DR, Hosker JP, Rudenski AS et al. Homeostasis model assessment: insulin resistance and beta-cell function from fasting plasma glucose and insulin concentrations in man. Diabetologia 28, 412-419 (1985). 
31 Expert Panel on Detection, Evaluation, and Treatment of High Blood Cholesterol in Adults. Executive Summary of the Third Report of the National Cholesterol Education Program (NCEP) Expert Panel on Detection, Evaluation, And Treatment of High Blood Cholesterol In Adults (Adult Treatment Panel III). JAMA 285, 2486-2497 (2001).

32 Bershtein LM. [Excretion of classical estrogens and total phenolsteroids in uterine body cancer patients]. Vopr. Onkol. 13(9), 48-52 (1967).

33 Webb PM. Obesity and gynecologic cancer etiology and survival. Am. Soc. Clin. Oncol. Educ. Book 2013,e222 (2013).

34 McClintock MK, Conzen SD, Gehlert S, Masi C, Olopade F. Mammary cancer and social interactions: identifying multiple environments that regulate gene expression throughout the life span. J. Gerontol. B Psychol. Sci. Soc. Sci. 60(Spec No 1), 32-41 (2005).

35 Bianchini F, Kaaks R, Vainio H. Overweight, obesity, and cancer risk. Lancet Oncol. 3(9), 565-574 (2002).

36 Esposito K, Chiodini P, Capuano A, Bellastella G, Maiorino MI, Giugliano D. Metabolic syndrome and endometrial cancer: a meta-analysis. Endocrine 45(1), 28-36 (2014).

37 Kassi E, Pervanidou P, Kaltsas G, Chrousos G. Metabolic syndrome: definitions and controversies. BMC Med. 9, 48 (2011).

38 Berthelsen $\mathrm{H}$, Svane H. Cancer of the endometrium and hyperestrinism; two different modes of origin of endometrial cancer. Dan. Med. Bull. 3(8), 236-239 (1956).

39 Brinton LA, Felix AS, McMeekin DS et al. Etiologic heterogeneity in endometrial cancer: evidence from a gynecologic oncology group trial. Gynecol. Oncol. 129(2), 277-284 (2013).

40 Danubio ME, Sanna E. Secular changes in human biological variables in western countries: an updated review and synthesis. J. Anthropol. Sci. 86, 91-112 (2008).

41 Huang CY, Chen CA, Chen YL et al. Nationwide surveillance in uterine cancer: survival analysis and the importance of birth cohort: 30冈year population-based registry in Taiwan. PLoS ONE 7(12), e51372 (2012).

42 Sherman ME. Theories of endometrial carcinogenesis: a multidisciplinary approach. Mod. Pathol. 13(3), 295-308 (2000).

43 Mulasi U, Kuchnia AJ, Cole AJ, Earthman CP. Bioimpedance at the bedside: current applications, limitations, and opportunities. Nutr. Clin. Pract. 30 (2), 180-193 (2015).

44 Phillips CM. Metabolically healthy obesity: definitions, determinants and clinical implications. Rev. Endocr. Metab. Disord. 14, 219-227 (2013).

- Interesting and important paper underlining the need of elaboration of standardized metabolically healthy obesity definitions.

45 Bell J, Hamer M, Sabia $S$ et al. The natural course of healthy obesity over 20 years. J. Am. Coll. Cardiol. 65(1), 101-102 (2015).

46 Blüher M. Are metabolically healthy obese individuals really healthy? Eur. J. Endocrinol. 171(6), R209-R219 (2014).
47 Berstein LM, Vasilyev DA, Berlev IV, Poroshina TE. Metabolically healthy obesity: changing prevalence in endometrial cancer and relation with clinical features of this tumor. Presented at: ENDO 97th Annual Meeting. San Diego, CA, USA, 5-8 March 2015 (Abstract THR-377).

48 Zhang Y, Liu L, Li C, Ai H. Correlation analysis between the expressions of leptin and its receptor (ObR) and clinicopathology in endometrial cancer. Cancer Biomark. 14(5), 353-359 (2014).

49 Yamauchi N, Takazawa Y, Maeda D et al. Expression levels of adiponectin receptors are decreased in human endometrial adenocarcinoma tissues. Int. J. Gynecol. Pathol. 31(4), 352-357 (2012).

50 Dossus L, Lukanova A, Rinaldi S et al. Hormonal, metabolic, and inflammatory profiles and endometrial cancer risk within the EPIC cohort - a factor analysis. Am. J. Epidemiol. 177(8), P787-P799 (2013).

51 Patel SM, Ratcliffe SJ, Reilly MP et al. Higher serum testosterone concentration in older women is associated with insulin resistance, metabolic syndrome, and cardiovascular disease. J. Clin. Endocrinol. Metab. 94(12), 4776-4784 (2009).

52 Collins L, Mohammed N, Ahmad T, Basaria S. Androgen deprivation therapy for prostate cancer: implications for cardiometabolic clinical care. J. Endocrinol. Invest. 35(3), 332-339 (2012).

53 Everett E, Tamimi H, Greer B et al. The effect of body mass index on clinical/pathologic features, surgical morbidity, and outcome in patients with endometrial cancer. Gynecol. Oncol. 90(1), 150-157 (2003).

54 Ortega-Molina A, Efeyan A, Lopez-Guadamillas E et al. Pten positively regulates brown adipose function, energy expenditure, and longevity. Cell. Metab. 15(3), 382-394 (2012).

55 Nedergaard J, Cannon B. The changed metabolic world with human brown adipose tissue: therapeutic visions. Cell. Metab. 11(4), 268-272 (2010).

56 Cypess AM, Kahn CR. Brown fat as a therapy for obesity and diabetes. Curr. Opin. Endocrinol. Diabetes Obes. 17(2), 143-149 (2010).

57 Spiegelman BM. Banting Lecture 2012: regulation of adipogenesis: toward new therapeutics for metabolic disease. Diabetes 62(6), 1774-1782 (2013).

58 Gesta S, Tseng YH, Kahn CR. Developmental origin of fat: tracking obesity to its source. Cell 131(2), 242-256 (2007).

59 Linkov F, Kokai L, Edwards R et al. The role of adiposederived stem cells in endometrial cancer proliferation. Scand. J. Clin. Lab. Invest. Suppl. 244, 54-58 (2014).

60 Cancer Genome Atlas Research Network, Kandoth C, Schultz $\mathrm{N}$ et al. Integrated genomic characterization of endometrial carcinoma. Nature 497(7447), 67-73 (2013).

-. Seminal paper showing possible existence of greater number of endometrial cancer types than it is believed at present.

61 Carlson MJ, Thiel KW, Yang S, Leslie KK. Catch it before it kills: progesterone, obesity, and the prevention of endometrial cancer. Discov. Med. 14(76), 215-22 (2012). 